\title{
A computer-linked runway for real time operation'
}

IAN G. MICHOLLS

UNIVERSITY OF WESTERN AUSTRALIA

This paper outlines a system for recording the running times of rats in a straight nuwway using a time-shared computer. A description is given of the hardware and software used, and the advantages of the system are discussed.

In a recent report on the use of computers for process control in psychological research, Fliege (1966) stated that one of the limitations of this type of application lies in the extremely high cost of computer time. With the advent of time-sharing facilities, however, costs can be drastically reduced by using the central processor only during the time actually needed for computation. In the case of an on-line device such as described below, the computation time demanded from the central processor during an experimental run of $8 \mathrm{hr}$. can be of the order of 2 or $3 \mathrm{sec}$.

It is the purpose of this paper to describe a realtime application of time-shared computer facilities to the measurement of running speed in a straight runway.

(1) The Runway. The runway, made of $1 / 2-\mathrm{in}$. plywood, is $13 \mathrm{ft}$. long, $4 \mathrm{in}$. high, and $2-1 / 2 \mathrm{in}$. wide. The start and goal boxes consist of the first and last 1-ft. sections of the runway and are separated from it by clear Plexiglas doors. The apparatus is covered by hinged Plexiglas lids. Photocells and lamps are positioned at 1-ft. intervals along the munway. Each time a new rat is placed in the runway, $E$ presses a button which sends a pulse (identification pulse) to the computer interface (see below) along a pair of telephone lines. The movement of a rat along the runway causes each photobeam to be interrupted and a pulse (timing pulse) to be sent to the computer interface along a second pair of telephone lines.

(2) The Computer. A PDP-6 computer is used for the data acquisition and processing. This machine (Digital Equipment Corporation, 1965a) has a 36-bit arithmetic processor with 16 fast accumulators, 15 index registers, built-in floating point arithmetic, and byte operation hardware. Memory protection and relocation registers are included for time sharing operations. Any input/output (I/O) device communicates with the processor by using the seven-channel hardware priority interrupt system. The computer can request data from an I/O device by setting a BUSY flag in the control register of the interface. When the data is supplied by the device a DONE flag is set which energizes one of the priority interrupt levels. (This is preset by software at monitor assembly time.) The computer, sensing the interrupt, services

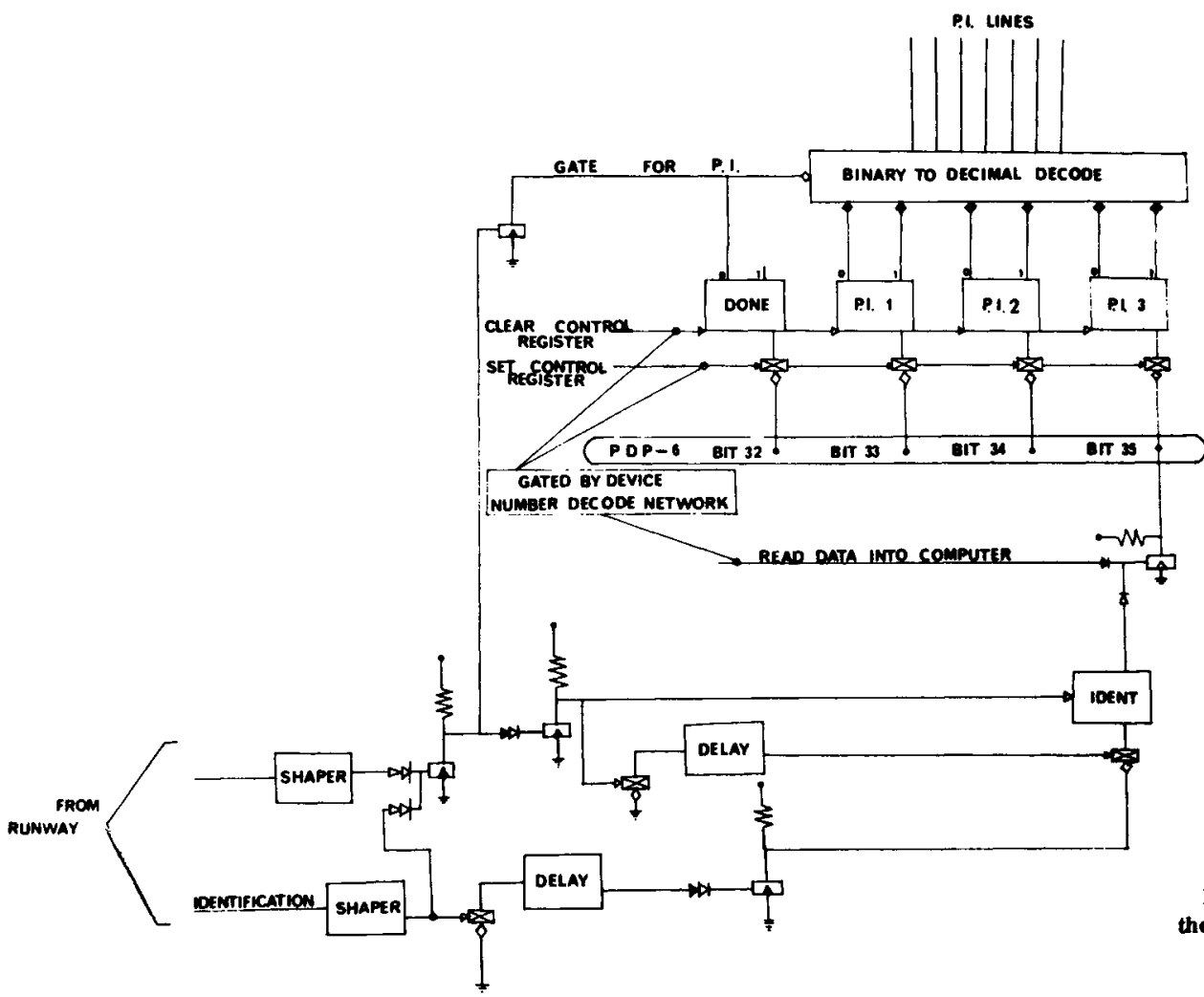

Fig. 1. Simplified diagram of the runway interface. 
the request and sets the BUSY flag to request more data. All devices are coupled to a single I/O bus and a particular interface is addressed by placing a device number on separate lines of the bus. Each interface has logic to decode its own device number.

(3) The Interface. Figure 1 shows a simplified diagram of the runway interface. Technically, only the pair of input lines supplying the timing pulses would be sufficient. Each rat could then be identified by counting the appropriate number of pulses. However, should a photocell fail, or, as is often the case churing extinction, should a rat not complete the course, this system of identification would not be possible. The second pair of lines (Identification lines) was added for this reason. The input signals are sharpened with Schmitt triggers and then fed to pulse shaping monostables. If the input signal is that for a new rat, the data flip flop is also set. Both inputs are "or-ed" together to set the DONE flag and enable the priority interrupt.

The device dependent monitor routine which services the interface reads the time from a real time clock and stores it in the user's area.

(4) The Real Time Clock. A 12-bit hardware clock with no carry propagation delay, constructed from DEC FLIPCHIPS, runs from a crystal (10 K c/s) and causes an interrupt approximately twice each second. A 36-bit software register is incremented by one on each interrupt.

The runway monitor service routine accesses the hardware real time clock and adds the contents of the appropriately shifted software register before storing in the user's buffer area. Provision is also built in to prevent the clock from trying to add one while it is being referenced by the monitor.

(5) The Monitor Service Routine. The FDP-6 time shared monitor (Digital Equipment Corporation, 1965b) provides the overall coordination and control of the total operating system handling all the I/O and carrying out the error detection necessary in a time shared gystem. Its modular construction easily allowed the addition of the runway interface service routine. This routine stores the time when an interrupt occurs, noting whether it was caused by an identification or a timing pulse.

As the runway is not given the highest priority of all I/O devices, the reaction time (or time between the occurrence of a signal external to the computer and the commencement of execution of the first useful instruction requested by the external signal) can vary depending on the interrupts of higher priority that occur during normal timesharing operation. If all higher interrupts were being used, the time variation would be $0.00004 \mathrm{sec}$. Thus the effect of time sharing on computer accuracy is negligible.

(6) The User.Program. This is a program which uses only 1000 words of core and is written in Macro language to initialize the interface and write the data supplied by the service routine on DECTAPE for later editing and analysis.

(7) Normal Operation Mode. The pulses from the runway can be fed directly into the computer for on-line running, or they can be recorded on an ordinary Phillips Stereotape recorder (one channel for time pulses and one for identification pulses) which is used as a buffer store and later played back into the same interface for off-line operation. Playback can be at eight times the recording speed thus taking $1 \mathrm{hr}$. to analyze $8 \mathrm{hr}$. of tape recording. Tests were carried out to measure the variation in pulse arrival times due to tape and recorder variations by replaying the same tape several times. Agreement between pulse intervals was better than $.01 \%$ which is well within other experimental errors.

(8) Advantages of the System.The major advantage of this system lies in the time saved in the automatic recording of the data. A recent experiment included 10 groups of 15 rats, each given four trials per day for 40 days. Since 13 running times were recorded on each trial, a total of 7800 times were recorded each day and 312,000 during the whole experiment. Obviously, an experiment of this kind could not have been possible without automation. It has been our experience that, using a system of this nature, experimental time can be halved even with a munway having as few as two or three timing signals.

The total cost of interfacing and timing equipment was approximately $\$ 750.00$, which is considerably below the cost of automating the recording system by any other means. The cost of computer time, assuming a time-sharing system, is relatively low since, as noted earlier, the ratio of experimental time to central processor time is approximately 10,000 to 1 .

An added advantage lies in increased reliability since errors in recording and transcribing data by hand are eliminated.

This system has been in use for 12 months with no malfunction.

\section{References}

Digital Equipment Comoration. The programmed data processor6 handbook. Maynard, Mass., 1965a.

Digital Equipment Corporation. $P D P-6$ multiprogramming system manual. Maynard, Mass., 1965b.

Fliege, S. Digital computers. In J. B. Sidowski (Ed.), Experimental methods and instrumentation in psychology. New York: McGrawHill, 1966.

\section{Note}

1. This work was sponsored by the Air Force Office of Scientific Research, Office of Aerospace Research, United States Air Force, under AFOSR Grant AF-AFOSR-968-66 to Vincent Di Lollo. The author is grateful to Dr. Di Lollo for his support and encouragement. 